\title{
SCANDINAVIAN JOURNAL OF MEDICINE \& SCIENCE IN SPORTS
}

\section{Variable, but not free-weight, resistance exercise potentiates jump performance following a task-specific warm-up.}

\begin{tabular}{|r|l|}
\hline Journal: & Scandinavian Journal of Medicine and Science in Sports \\
\hline Manuscript ID & SJMSS-O-716-18.R2 \\
\hline Manuscript Type: & Original Article \\
\hline Author: & 07-Nov-2018 \\
\hline Complete List of Authors: & $\begin{array}{l}\text { Mina, Minas; University of Derby, School of Sport, Outdoor and Exercise } \\
\text { Science } \\
\text { Blazevich, Anthony; Edith Cowan University, Centre for Exercise and } \\
\text { Sports Science Research } \\
\text { Tsatalas, Themistoklis; University of Thessaly, Department of Physical } \\
\text { Education and Sport Science } \\
\text { Giakas, Giannis; University of Thessaly, Department of Physical } \\
\text { Education and Sport Science } \\
\text { Seitz, Laurent; Edith Cowan University, Centre for Exercise and Sports } \\
\text { Science Research } \\
\text { Kay, Anthony; The University of Northampton, Sport, Exercise \& Life } \\
\text { Sciences }\end{array}$ \\
\hline Keywords: & $\begin{array}{l}\text { elastic bands, PAP, conditioning activity, explosive strength, kinetics, } \\
\text { kinematics }\end{array}$ \\
\hline
\end{tabular}

\section{SCHOLARONE Manuscripts}


2 Variable, but not free-weight, resistance back squat exercise potentiates jump performance following a comprehensive task-specific warm-up

\section{AUTHORS}

5 Minas A. Mina ${ }^{1}$, Anthony J. Blazevich², Themistoklis Tsatalas ${ }^{3}$, Giannis Giakas ${ }^{3}$, Laurent B.

$6 \quad$ Seitz $^{2} \&$ Anthony D. Kay ${ }^{4}$

7

\section{INSTITUTIONAL AFFILIATIONS}

$9 \quad{ }^{1}$ Sport, Outdoor and Exercise Science, University of Derby, Derby, United Kingdom

$10 \quad{ }^{2}$ Centre for Exercise and Sports Science Research, School of Medical \& Health Sciences, Edith

11 Cowan University, Joondalup, Western Australia

$12 \quad{ }^{3}$ Department of Physical Education \& Sport Science, University of Thessaly, Greece

${ }^{4}$ Sport, Exercise \& Life Sciences, University of Northampton, Northampton, United Kingdom

\section{RUNNING HEAD}

16 Elastic band use improves jump performance

\section{CORRESPONDING AUTHOR}

19 Minas A. Mina ${ }^{1}$

20 Sport, Outdoor and Exercise Science

21 University of Derby

22 Kedleston Road, Derby

23 DE22 1GB, United Kingdom.

24 Tel: $+44(0) 7774014822$.

25 Email: M.Mina@derby.ac.uk 


\section{ABSTRACT}

Studies examining acute, high-speed movement performance enhancement following intense muscular contractions (frequently called 'post-activation potentiation'; PAP) often impose a limited warm-up, compromizing external validity. In the present study the effects on countermovement vertical jump (CMJ) performance of back squat exercises performed with or without elastic bands during warm-up were compared. After familiarization, fifteen active men visited the laboratory on two occasions under randomized, counterbalanced experimental squat warm-up conditions: (1) free-weight resistance (FWR) and (2) variable resistance (VR). After completing a comprehensive task-specific warm-up, three maximal CMJs were performed followed by three back squat repetitions completed at $85 \%$ of 1-RM using either FWR or VR. Three CMJs were then performed $30 \mathrm{~s}, 4 \mathrm{~min}, 8 \mathrm{~min}$ and 12 min later. During CMJ trials, hip, knee and ankle joint kinematics, ground reaction force data and vastus medialis, vastus lateralis and gluteus maximus electromyograms (EMG) were recorded simultaneously using 3D motion analysis, force platform, and EMG techniques, respectively. No change in any variable occurred after FWR $(p>0.05)$. Significant increases $(p<0.05)$ were detected at all time points following VR in CMJ height (5.3-6.5\%), peak power (4.4-5.9\%), rate of force development (12.9-19.1\%), peak concentric knee angular velocity (3.1-4.1\%) and mean concentric vastus lateralis EMG activity (27.5-33.4\%). The lack of effect of the free-weight conditioning contractions suggests that the comprehensive task-specific warm-up routine mitigated any further performance augmentation. However, the improved CMJ performance following the use of elastic bands is indicative that specific alterations in force-time properties of warm-up exercises may further improve performance.

Keywords: elastic bands, PAP, conditioning activity, explosive strength, kinetics, kinematics 


\section{INTRODUCTION}

52 Pre-exercise (i.e. warm-up) routines are typically designed to precondition the neuromuscular system to enhance performance and reduce injury risk during subsequent high-intensity 54 physical activities. ${ }^{1-3}$ Performing maximal or near-maximal muscular contractions during a 55 warm-up routine are important as they can induce short-term increases in force production and 56 physical performance ${ }^{4}$ through a number of mechanisms including, but not limited to, increases 57 in muscle temperature, ${ }^{5}$ reductions in muscle thixotropy or viscosity, ${ }^{3}$ increases in myofilament calcium sensitivity, ${ }^{6}$ an increased neural drive (leading to higher-frequency motor unit discharge) and optimization of motor control strategies. ${ }^{7}$ Such changes lead to an increased mechanical power output (i.e. above previous maximal voluntary capacity), a state often referred to as post-activation potentiation (PAP) but which may not be synonymous with 'classic' PAP, which refers to an increase in muscular force production during an electrically elicited (twitch) contraction. ${ }^{8}$ Regardless of the mechanism, short-term improvements in performance (i.e. post-activation performance enhancements [PAPE] $)^{9}$ are commonly reported following intense muscular contractions that have important implications for the design of warm-up strategies.

The acute augmentation of physical performance has been explored using different warm-up strategies including light muscle stretching, cycling, running and sub-maximal repetitions of the primary $\operatorname{task}^{10}$ or no warm-up at all. ${ }^{8}$ Consequently, a "comprehensive task-specific" warm-up (including progressively intense task-specific conditioning activities) is often not provided prior to the specific activity being tested. Although warm-up strategies adopted to potentiate muscular force production have been shown to enhance athletic performance following a conditioning activity, it is unclear whether the enhancement of athletic performance 
observed is a consequence of acute neuromuscular alteration relating to the conditioning activity, or whether it simply reflects a standard warm-up itself. ${ }^{11}$

Heavy resistance exercise has been shown to acutely potentiate muscle force output, at least when a comprehensive task-specific warm-up is not completed, ${ }^{2,8,12}$ however force production can also be reduced as a result of fatigue or coordination interference (i.e. perseveration) processes, which may mask any potentiating effects. ${ }^{1}$ Some studies have reported that vertical jump performance enhancements can be detected after only $20 \mathrm{~s}^{13}$ and $90 \mathrm{~s}^{14}$ following maximal isometric squats and heavy box squats, respectively. Findings from these studies are indicative that effects may be detected within the time course of "classic" PAP observed using muscle twitch examinations. ${ }^{8}$ Nonetheless, a meta-analysis of the literature revealed that minimal performance enhancement was likely when the rest period was less than 2 min, whereas longer rest periods of 3-7 min were more beneficial. ${ }^{15}$ The equivocal findings likely result from disparate study methodologies including types of conditioning activity (i.e. movement-pattern specificity), performance tasks, delay between the conditioning activity and performance testing, study participant characteristics (e.g. experienced/novice lifters) and warm-up performed, which limit our understanding of the potentiating effects of these warmup strategies.

The countermovement vertical jump (CMJ) task is commonly performed in sport but is also a model commonly used to test power and muscle function in clinical research environment. Various high-intensity exercise types have been performed before maximal CMJ tests including resistance-, plyometric-, and electrical muscle stimulation-based exercises. ${ }^{16,18}$ The back squat exercise is a fundamental exercise for the development of lower-limb strength and power ${ }^{12}$ and its use during a warm-up has been reported to improve subsequent functional 
100 performance including CMJ height; ${ }^{1,4}$ this enhancement is commonly attributed to the PAP 101 effect. However, maximal voluntary muscle activity occurs only during a short period in the early ascending (concentric) phase, near the "sticking point" in successful maximal (1-RM) back squat attempts. The larger internal and smaller external moment arms developed at the hip and knee joints (resulting in a greater mechanical advantage) combined with the optimized force-length characteristics of lower-limb muscles, ensures that only a submaximal muscle activation is needed for successful completion of the remaining part of the lift. ${ }^{18}$ Thus, theoretically, variations of the exercise that evoke a greater muscle activation throughout the lift could result in a greater warm-up (i.e. PAPE) effect and improve CMJ performance. A possible means to alter the loading characteristics of the squat lift is the use of elastic bands to reduce the external load in the deepest part of the squat while increasing external load when the joints are more extended, the internal moment arms are greater and optimal muscle lengths are achieved. ${ }^{18,19}$ Previous studies comparing elastic bands to free-weight squats for muscle activities (EMG), kinematics and kinetics has shown significantly higher EMG, movement velocity, and external power in the first quarter of the eccentric phase and the last quarter of the concentric phase of the squat exercise when using elastic bands. ${ }^{19}$ Accordingly, it has been found that preconditioning contractions using elastic bands significantly increased subsequent 1-RM squat test performance without detectable changes in knee extensor muscle activity or knee flexion angle, although eccentric and concentric velocities were reduced. ${ }^{2}$

Accordingly, elastic bands can be used to increase resistance in ranges of motion where the muscles can produce the greatest relative force as well as unload the system where muscle forces are compromized, and thus allow a larger overall impulse to be produced. Given the possibility for higher muscle activation and greater total work done during the lift, it might be hypothesized that these conditions would allow for a greater potentiating effect. 
126 Individuals incorporating the use of elastic band-based strategies into a warm-up routine may 127 observe an acute enhancement of performance, and thus benefit from a greater mechanical 128 stimulus during training. ${ }^{2}$ However, a common limitation in the literature is that minimal or 129 no warm-up has been provided before imposing the conditioning activity $^{8}$ limiting the 130 comprehensive applicability and external validity of the data. Therefore, the purpose of this 131 study was to compare the influence of free-weight resistance (FWR) and variable resistance 132 (VR; imposed through elastic bands) squat exercises following a task-specific comprehensive 133 warm-up on subsequent $\mathrm{CMJ}$ performance at different post-conditioning time points (i.e. $30 \mathrm{~s}$, $1344 \mathrm{~min}, 8 \mathrm{~min}$, and $12 \mathrm{~min}$ ). It was hypothesized that (i) FWR and VR would enhance 135 subsequent CMJ performance; however the variation in resistance imposed by the elastic bands 136 during the squat lift would (ii) further enhance subsequent CMJ performance, (iii) alter CMJ 137 kinetic and kinematic parameters (i.e. peak power, peak eccentric kinetic energy, impulse- and 138 time-based descent-to-ascent asymmetry indexes, vertical stiffness $\left(\mathrm{K}_{\mathrm{ver}}\right)$, rate of force 139 development (RFD), hip, knee and ankle joint kinematics), and (iv) increase the muscle activity 140 of the lower-limb extensor muscles more than squatting without elastic bands.

\section{MATERIALS AND METHODS}

\section{Participants}

144 Fifteen active men (age $=21.7 \pm 1.1 \mathrm{y}$, height $=1.8 \pm 1.9 \mathrm{~m}$, mass $=77.6 \pm 2.6 \mathrm{~kg})$ with $\geq 5 \mathrm{y}$ experience with heavy weight training of varying levels (from regional to elite) and training backgrounds volunteered to participate after providing written informed consent and completing a pre-test medical questionnaire. The participants' training protocols involved resistance training, sprint running, power exercises, dynamic/explosive exercises, agility drills and other specific exercises relevant to their sports. The participants had no recent illness or 
150

151

152

153

154

155

156

157

158

159

160

161

162

163

164

165

166

167

168

169

170

171

172

173

174

lower-limb injury, were instructed to maintain normal eating and drinking habits throughout the study, and avoided strenuous exercise and stimulant use at least $48 \mathrm{~h}$ prior to testing. Ethical approval was granted by the ethics committee at the University of Thessaly, Greece, with the study conducted in accordance with the Declaration of Helsinki. Effect size (ES) values (Cohen's $d$ ) were calculated from mean changes in variables (jump height, power, RFD and EMG) from previous studies using similar methods. To ensure an adequate population to reach statistical power (set at 0.8 ) was recruited, effect sizes were initially calculated from related $\operatorname{research}^{21-23}$ for jump height $(\mathrm{ES}=1.48)$, power $(\mathrm{ES}=1.0), \mathrm{RFD}(\mathrm{ES}=1.29)$, and EMG $(\mathrm{ES}=$ 1.2). To ensure an adequate sample, the measure with the smallest ES (power, 1.0) was used to calculate sample size. The analysis revealed that the initial sample size required for statistical power was 14; thus, considering the possibility of participant withdrawal and data loss, 18 participants were recruited with 15 participants completing the study.

\section{Protocol}

\section{Overview}

A randomized, cross-over design was implemented to compare CMJ performance following two warm-up conditions: free-weight resistance (FWR) or variable resistance (VR) back squat exercise. Participants completed a familiarization session one week prior to the two experimental sessions, each separated by $72 \mathrm{~h}$ and performed at the same time of the day. During familiarization, anthropometric characteristics were recorded, one-repetition maximum (1-RM) back squat load was determined, and the participants were familiarized with all experimental procedures. During experimental conditions, following the comprehensive taskspecific warm-up (described later), the participants performed three pre-intervention CMJs followed by back squats at $85 \%$ of $1-\mathrm{RM}$ using either FWR or VR warm-up. CMJ trials were then performed at $30 \mathrm{~s}, 4 \mathrm{~min}, 8 \mathrm{~min}$ and $12 \mathrm{~min}$ after the intervention. Peak power output, 
peak eccentric kinetic energy, impulse- and time-based descent-to-ascent asymmetry indexes, peak normalized (to body weight) $\mathrm{K}_{\text {vert }}$ and RFD, peak knee flexion angle, peak eccentric and concentric knee angular velocities, peak and mean eccentric and concentric electromyograms (EMG; vastus lateralis (VL), vastus medialis (VM), gluteus maximus (Glut)), and jump height were measured during all $\mathrm{CMJ}$ trials (described later).

\section{Familiarization session and one-repetition (1-RM) squat lift test}

The 1-RM back squat protocol was adopted from Sheppard and Triplett et al. ${ }^{24}$ Participants initially performed a 5 min cycling warm-up (Monark 874E, Varberg, Sweden) at $65 \mathrm{rpm}$ with a 1-kg resistance load followed 2 min later by 2 sets of 10 back squat repetitions using an unloaded 20-kg Olympic bar. The participants then completed 8-10 repetitions of the squat lift exercise at $50 \%$ of their estimated 1-RM load before the load was increased by $20 \%$ for $3-5$ repetitions, and by a further $20 \%$ for $2-3$ repetitions with a 2 min rest between sets. The load was finally increased by $5 \%$ movements with $2-4$ min rest between lifts until participants failed to complete the lift; the previous successful attempt was recorded as their 1-RM load. To ensure correct technique, participants were instructed to place the bar above the posterior deltoids at the base of the neck and position the feet shoulder width apart with the toes pointed slightly outward and attempt to squat to a position where the knee was flexed to $\sim 90^{\circ}$ before returning to a standing position. This was visually assessed by an experienced, certified British Amateur Weight Lifting Association (BAWLA) spotter throughout all testing procedures to ensure correct technique and safety during the lifts, with participants receiving strong verbal encouragement to promote maximal effort.

\section{Comprehensive task-specific warm-up and countermovement jump trials}


199 During the experimental trials the participants performed a comprehensive task-specific warm200 up consisting of 5 min of cycling followed by five continuous unloaded squats (i.e. non201 jumping) at a rhythm of $2 \mathrm{~s} / 2 \mathrm{~s}$ (eccentric/concentric) and a further 5 squats at a rhythm of 1 202 s/ 1 s after a 30 s rest. After 20 -s rest, five continuous CMJs were performed at $\sim 70 \%$ of the 203 participants' perceived maximum and, after a further 30 s rest, maximal CMJs were performed 204 every $30 \mathrm{~s}$ until three consecutive jumps were within $3 \%$ of jump height (4-7 jumps were 205 performed in all trials). The CMJ was performed from a stationary upright standing position 206 with hands positioned on the hips, making a preliminary downward movement with the hips 207 and knees flexed, and immediately jumping vertically up as high possible. ${ }^{12}$

Two minutes after the completion of the warm-up, three maximal pre-intervention CMJ trials were performed to establish baseline (i.e. after warm-up) performance. A conditioning set of three repetitions of back squats at $85 \%$ of the previously determined 1-RM using either FWR or VR (described later) was then performed before the participants completed three CMJs 30 s, 4 min, 8 min and $12 \mathrm{~min}$ (see Table 1) later with participants receiving verbal encouragement to jump as high as possible. The post-intervention intervals were selected from previous data describing the time-course of the performance augmentation (PAP) response..$^{21,25}$

\section{Conditioning activities: free-weight resistance (FWR) and variable resistance (VR)}

220 During the FWR condition, the load was adjusted to $85 \%$ of the previously determined 1-RM load with the participants performing one set of three-repetition back squats. In the VR warmup condition, $35 \%$ of the total load was generated from band resistance. To ensure a similar load of $85 \%$ 1-RM across FWR and VR conditions, mechanical properties of the bands were 
224

225

226

227

228

229

230

231

232

233

234

235

236

237

238

239

240

241

242

243

244

245

246

247

248

determined to allow the band resistance to generate $35 \%$ of the total load. Half of the band's resistance was subtracted from the total free-weight load to ensure the elastic bands did not have substantially different average resistance compared with the FWR condition, thus both the FWR and VR warm-up conditions were equalized, as previously reported..$^{20,22}$ The participants stood on a force platform with 85\% 1-RM load to determine the combined load $(\mathrm{kg})$, the bar was then unloaded to adjust the band tension. The elastic bands were anchored to the floor with custom-made weight stands and attached equidistant to the ends of the Olympic bar to ensure the participant's stability. The thickness and lengths of the elastic bands were adjusted so that: (i) the tension in the bands increased the ground reaction force (measured by force platform) by $35 \%$ of the $85 \%$ load when the participants were standing, but (ii) bands were slack in a full squatting position and thus provided no additional loading. The linear force-length properties of the bands ensured, therefore, that the average load during the lift equated to $35 \%$ of the total load. For example, a $100-\mathrm{kg}$ load in the FWR condition would require $35-\mathrm{kg}(35 \%)$ to be generated from the bands. Half of the $35-\mathrm{kg}$ load (i.e. $17.5 \mathrm{~kg}$ ) was removed from the bar with the $35-\mathrm{kg}$ resistance added from the bands providing a total load of $117.5 \mathrm{~kg}$ in the standing position. As band tension reduced as the participant squats, $35 \mathrm{~kg}$ of load was removed leaving the $82.5 \mathrm{~kg}$ from the bar in the full squatted position. Thus, the average loading throughout the lift in this example is $100 \mathrm{~kg}$, identical to the FWR condition whilst enabling $35 \%$ to be generated by band tension.

\section{Kinetic and kinematic analyses}

Kinematic data were collected during the CMJs using a Vicon motion analysis system (TSeries, Oxford Metrics LTDA, Oxford, UK) with 10 cameras operating at $100 \mathrm{~Hz}$ surrounding two force platforms (Bertec, FP4060-10-2000, Bertec Corporation, Columbus, OH, USA). Ground reaction forces were sampled at $1000 \mathrm{~Hz}$ and time-synchronized with the Vicon system 
249 (see Figure 1). The data were then filtered using Woltring's quantic spline algorithm ${ }^{26}$ with a 250 mean squared error setting of 15 before running the Plug-In-Gait biomechanical model (Vicon 251 Plug-in-Gait, Oxford Metrics). The procedures identified by Davis et al. ${ }^{27}$ were followed to 252 define Cardan angles and to reconstruct a system of embedded coordinates from the marker set 253 to $0^{\circ}$ at the three joints of the lower extremities (hip, knee and ankle) in a standing position.

254 Lower-limb kinetic and kinematic data were captured by placing 16 reflective markers over the 255 pelvis, left and right thigh, left and right shank in a straight line, and the left and right foot at a 256 right angle to the leg. Data were analyzed using Vicon Nexus (v.2.3) software to determine 257 peak hip, knee and ankle flexion angle and angular velocity data during the pre- and post258 intervention CMJ trials (see Figure 1).

Figure 1 about here

All jumps were performed from the standing position with each foot in parallel on two force platforms providing a separate yet time-synchronized measurement of the force data for each leg. The participant's body weight was calculated by averaging the vertical force from each platform when the participants were stationary. The initiation of the jump (i.e. the beginning of the eccentric phase) was identified as the point when the ground reaction force $(\mathrm{N})$ decreased 2 standard deviations (SD) below the mean baseline force. The vertical ground reaction force was integrated using the trapezoid method during the eccentric and concentric phases of the jump. The net impulse was calculated independently and summed from the left and right force platforms. Ground reaction forces were directly quantified by integrating the applied force over time (i.e. impulse), which is equivalent to the change in momentum of the body:

$$
J=\int F d t=\Delta p
$$

273 where $J=$ impulse, $F=$ force, $t=$ time and $\Delta p=$ change in momentum. 
275 The take-off velocity was determined from impulse by dividing by body mass, and the jump

276 height was calculated using standard equations for motion. ${ }^{28}$ To calculate power, the impulse-

277 momentum approach was used. Since the force, mass and initial velocity conditions were

278 known, instantaneous velocity could be calculated. The instantaneous power was calculated

279 as force $\times$ velocity and the peak values were determined for the propulsive phase of the CMJ:

280

281

282

283

284

285

286

287

288

289

290

291

292

293

294

295

296

297

298

$$
\begin{gathered}
V_{(0)}=0 \\
F(\mathrm{i}) t=m\left(v_{(\mathrm{i}+1)}-v_{(\mathrm{i})}\right) \\
\Delta v=\left(F_{(\mathrm{i})} t\right) / m \\
P_{(\mathrm{i})}=F_{(\mathrm{i})} \times V_{(\mathrm{i})}
\end{gathered}
$$

where $F=$ force, $t=1 /$ sampling frequency, $m=$ mass of body, load, $v=$ velocity, and $P=$ power.

The peak eccentric kinetic energy (KE) developed during the jumps was calculated as:

$$
K E=1 / 2 m v^{2}
$$

where $m$ is the participant's mass and $v$ is the velocity of the countermovement phase.

The impulse-based asymmetry index was calculated by dividing the negative and positive impulses, where the negative impulse describes the impulse that negatively accelerates the body downwards and the positive impulse accelerates the body upwards. The index was calculated to estimate the efficiency of the metabolic energy conversion into mechanical work (i.e. storage of elastic energy during eccentric contraction) performed during the $\mathrm{CMJ}$ from the force applied by the body to the ground ${ }^{29}$ and subsequently released energy during the concentric phase of the SSC. The time-based asymmetry index was calculated as the quotient of times $\mathrm{A}+\mathrm{B}$, where $A$ is the time from force first rising above 1 body weight to the peak 
299 vertical force and $B$ is the time from peak force until force drops below 1 body weight. $\mathrm{K}_{\text {vert }}$ 300 was calculated by dividing the peak vertical ground reaction force by the maximal vertical 301 displacement of the center of mass during contact with the ground ${ }^{30}$

$$
K_{\text {vert }}=F_{\max } / \Delta y
$$

where $\mathrm{F}_{\max }=$ maximum vertical force, and $\Delta \mathrm{y}=$ maximum vertical displacement of the center of mass. The vertical displacement was determined by the double integration of the vertical force trace according to methods of Cavagna. ${ }^{31}$

The peak RFD (normalized to body weight) was calculated from the initiation of the jump (i.e. first rise in force during the eccentric phase) using the average force-time curve with a 50-ms time window.

310

Muscle activity (electromyography; EMG)

312 EMG data were collected wirelessly using a Myon MA-320 EMG system (Myon AG, 313 Schwarzenberg, Switzerland) from vastus lateralis (VL), vastus medialis (VM) and gluteus 314 maximus (Glut). The skin was shaved, abraded and cleansed with alcohol before bipolar 315 adhesive surface electrodes (Noraxon Dual Electrodes, Ag-AgCl, Noraxon USA, Inc, 316 Scottsdale, AZ) were placed over the muscle belly with an inter-electrode distance of $2 \mathrm{~cm}$ 317 according to SENIAM guidelines. EMG data were sampled at $2000 \mathrm{~Hz}$ and imported into 318 ProEMG software (version 4.1) and filtered using a Butterworth (20-500 Hz bandpass) filter 319 before using a symmetric moving root-mean-square algorithm with a 50-ms sampling window. 320 The Myon EMG software was integrated with an optimal tracking device for synchronization 321 between the systems (Vicon motion analysis system, Oxford, UK). The normalized EMG 322 amplitude during isometric squat lifts (\% maximal voluntary contraction [MVC]) for each 
323

324

325

326

327

328

329

330

331

332

333

334

335

336

337

338

339

340

341

342

343

344

345

346

347

muscle was used as a measure of neuromuscular activity during the jumps (see Figure 1), with peak and mean EMG activity recorded during the eccentric and concentric phases.

\section{Data analyses}

All data were analyzed using SPSS statistical software (version 24.0; IBM, Chicago, IL, USA); all data are presented as mean \pm SE. Normal distribution was assessed using Shapiro-Wilks test; no significant difference $(p>0.05)$ was detected in any variable indicating that all data sets were normally distributed. Separate multivariate analyses of variance (MANOVAs) were used to compare (a) jump height and peak power, and (b) EMG. Where significant differences were detected, separate two-way repeated measures ANOVAs (time $\times$ condition) were used to determine differences in (a) jump height, (b) peak power, (c) peak eccentric kinetic energy, (d) impulse- and time-based descent-to-ascent asymmetry indexes, (e) peak normalized RFD, (f) peak hip, knee and ankle flexion angle, (g) peak eccentric and concentric hip, knee and ankle angular velocities, (h) peak and mean eccentric and concentric EMG activities during CMJ trials. Significance was accepted at $p<0.05$ for all tests.

\section{Reliability}

Reliability for all measures was determined during the pre-intervention vertical jumps from the VR and FWR warm-up conditions. No significant differences $(p>0.05)$ were detected in any measure and high intraclass correlation coefficients (ICCs) calculated for jump height (0.95), peak power (0.98), peak eccentric kinetic energy (0.99), impulse- $(0.96)$ and time-based $(0.91)$ asymmetry indexes, $\mathrm{K}_{\mathrm{vert}}(0.81)$, peak RFD to $50 \mathrm{~ms}(0.92)$, peak hip, knee and ankle flexion angle ranged from 0.67 to 0.96 , peak angular velocities ranged from $0.76,0.95,0.85$ to 0.85 , 0.950 .79 for hip, knee and ankle, respectively. ICCs for the EMG data ranged from 0.73 to $0.89,0.85$ to $0.92,0.85$ to 0.92 for VL, VM and Glut, respectively. Coefficients of variation 
$348(\mathrm{CoV})$ expressed as a percentage of the mean were also calculated for jump height (8.0\%), peak 349 power (6.2\%), peak eccentric kinetic energy (8.5\%), impulse- $(4.9 \%)$, and time-based (14.6\%) 350 asymmetry indexes, $\mathrm{K}_{\mathrm{vert}}(8.7 \%)$, peak RFD to $50 \mathrm{~ms}(12.5 \%)$, peak hip, knee and ankle flexion 351 angle ranged from $3.8 \%$ to $7.6 \%$, peak angular velocities ranged from $5.6 \%, 5.2 \%, 14.8 \%$ to $3525.0 \%, 3.4 \%, 6.3 \%$ for hip, knee and ankle, respectively. CoVs for EMG data 9.0\% to 14.3\%, $35311.3 \%$ to $14.1 \%, 14.9 \%$ to $22 \%$ for VL, VM and Glut, respectively.

\section{RESULTS}

In the FWR condition, no significant changes $(p>0.05)$ were found in jump height (range $=$ $3.0 \pm 2.0 \%$ to $4.9 \pm 2.2 \%$ ) at any time point compared with pre-intervention data (see Figure 1). Also, no significant changes $(p>0.05)$ were observed in peak power $(0.1 \pm 2.4 \%$ to $3.6 \pm$ $1.6 \%)$, peak eccentric kinetic energy $(0.5 \pm 4.6 \%$ to $4.9 \pm 3.6 \%)$, impulse- $(0.6 \pm 1.6 \%$ to $2.0 \pm$ $1.9 \%)$ and time-based $(4.5 \pm 7.0 \%$ to $14.8 \pm 8.4 \%)$ asymmetry indexes, $\mathrm{K}_{\mathrm{vert}}(3.1 \pm 4.3 \%$ to 5.8 $\pm 4.1 \%)$ or peak normalized RFD $(3.1 \pm 6.1 \%$ to $11.8 \pm 8.4 \%)$ at any time point (see Table 2$)$. No changes $(p>0.05)$ were detected in peak eccentric hip $(0.5 \pm 1.7 \%$ to $2.6 \pm 1.9 \%)$, knee $(0.5 \pm 2.1 \%$ to $2.6 \pm 2.2 \%)$, ankle $(2.2 \pm 5.4 \%$ to $9.0 \pm 5.0 \%)$ or concentric hip $(1.2 \pm 2.1 \%$ to $3.7 \pm 2.0 \%)$, knee $(0.5 \pm 1.7 \%$ to $1.7 \pm 2.0 \%)$, ankle $(1.4 \pm 2.1 \%$ to $4.7 \pm 2.6 \%)$ angular velocities, or peak hip $\left(1.5 \pm 1.3^{\circ}\right.$ to $\left.3.4 \pm 1.2^{\circ}\right)$, knee $\left(0.1 \pm 1.2^{\circ}\right.$ to $\left.1.7 \pm 1.9^{\circ}\right)$, ankle $(0.1 \pm$ $0.6^{\circ}$ to $0.6 \pm 0.5^{\circ}$ ) flexion angle (see Table 3). Furthermore, no changes in peak or mean eccentric EMG eccentric activity $(p>0.05)$ in $\mathrm{VL}($ peak $=2.4 \pm 3.8 \%$ to $7.2 \pm 5.2 \%$; mean $=$ $0.7 \pm 6.4 \%$ to $7.3 \pm 5.7 \%), \mathrm{VM}($ peak $=0.6 \pm 3.2 \%$ to $8.3 \pm 5.0 \%$; mean $=8.9 \pm 4.5 \%$ to 10.9 $\pm 3.2 \%$ ), Glut (peak $=0.9 \pm 6.0 \%$ to $8.7 \pm 3.8 \%$; mean $=2.3 \pm 6.0 \%$ to $10.7 \pm 7.4 \%$ ) or concentric $\mathrm{EMG}$ in VL (peak $=0.4 \pm 10.3 \%$ to $9.4 \pm 8.3 \%$; mean $=2.2 \pm 8.6 \%$ to $7.0 \pm 6.9 \%$ ),

$371 \mathrm{VM}($ peak $=0.5 \pm 4.6 \%$ to $7.1 \pm 5.2 \%$; mean $=1.2 \pm 7.1 \%$ to $9.5 \pm 5.4 \%)$ or Glut $($ peak $=1.3$ $372 \pm 5.7 \%$ to $10.4 \pm 5.6 \%$; mean $=2.1 \pm 6.6 \%$ to $8.3 \pm 8.9 \%$ ) were detected (see Table 4 ). 
Table 2 about here

In the VR condition, significant increases $(p<0.05)$ in CMJ height were detected at $30 \mathrm{~s}(5.9$ $\pm 1.2 \%), 4 \min (5.6 \pm 1.8 \%), 8 \min (6.5 \pm 2.6 \%)$ and $12 \min (5.3 \pm 2.5 \%)$ time points compared

with pre-intervention data (see Figure 2). Significant increases $(p<0.05)$ were also observed in peak power at $30 \mathrm{~s}(4.7 \pm 1.2 \%), 4 \min (5.9 \pm 1.3 \%), 8 \min (4.4 \pm 1.7 \%)$ and $12 \min (4.8 \pm$ 1.7\%) time points compared to pre-intervention data. These changes in CMJ height and power were also statistically different to FWR $(p<0.05)$. Similarly, significant increases $(p<0.05)$ were found in peak normalized RFD at $30 \mathrm{~s}(18.9 \pm 7.8 \%), 4 \min (12.9 \pm 5.9 \%), 8 \mathrm{~min}(19.1 \pm$ $5.0 \%)$ and $12 \min (16.0 \pm 8.1 \%)$ compared to pre-intervention data. However, no significant change $(p>0.05)$ in peak eccentric kinetic energy $(0.4 \pm 4.8 \%$ to $5.2 \pm 4.8 \%)$ or impulse- $(1.4$ $\pm 1.5 \%$ to $4.6 \pm 2.4 \%)$ or time-based $(7.4 \pm 11.7 \%$ to $13.0 \pm 12.5 \%)$ asymmetry indexes, $\mathrm{K}_{\text {vert }}$ $(6.6 \pm 4.5 \%$ to $8.9 \pm 3.7 \%)$ were found following the VR warm-up condition at any time point (see Table 2).

Table 3 about here

No significant change in peak hip $\left(1.3 \pm 1.3^{\circ}\right.$ to $\left.1.9 \pm 1.5^{\circ}\right)$, knee $\left(0.9 \pm 2.9^{\circ}\right.$ to $\left.4.1 \pm 3.0^{\circ}\right)$, ankle $\left(0.9 \pm 0.4^{\circ}\right.$ to $\left.1.4 \pm 0.7^{\circ}\right)$ flexion angles were observed in VR at any time point. Similarly, no changes $(p>0.05)$ were found at any time point (see Table 3$)$ in peak eccentric hip $(0.2 \pm$ $2.2 \%$ to $2.5 \pm 2.2 \%)$, knee $(0.04 \pm 1.7 \%$ to $2.6 \pm 2.5 \%)$, ankle $(0.1 \pm 6.6 \%$ to $6.7 \pm 8.7 \%)$ or or peak or mean eccentric EMG amplitudes for VL (peak $=0.5 \pm 4.3 \%$ to $3.1 \pm 4.3 \%$, mean $=$ 
398

399

400

401

402

403

404

405

406

407

408

409

410

411

412

413

414

415

416

\section{DISCUSSION}

418 The primary aim of the present study was to assess the magnitude and time-course of changes 419

420

421

422

$4.9 \pm 6.0 \%$ to $9.2 \pm 6.5 \%$ ), $\mathrm{VM}$ (peak $=2.1 \pm 4.6 \%$ to $9.6 \pm 4.0 \%$, mean $=4.9 \pm 5.4 \%$ to $6.7 \pm$ $5.8 \%$ ) or Glut (peak $=2.2 \pm 6.3 \%$ to $4.6 \pm 6.3 \%$, mean $=3.5 \pm 7.4 \%$ to $4.9 \pm 6.5 \%$ ). However, a significant increase $(p<0.05)$ was found in peak concentric knee angular velocity at $30 \mathrm{~s}(3.1$ $\pm 1.4 \%), 4 \min (4.1 \pm 1.7 \%), 8 \min (3.2 \pm 1.0 \%)$ and $12 \min (3.1 \pm 1.5 \%)$ and mean concentric VL EMG activity at $30 \mathrm{~s}(28.1 \pm 10.5 \%), 4 \mathrm{~min}(31.5 \pm 11.0 \%), 8 \min (33.4 \pm 15.9 \%)$ and 12 $\min (27.5 \pm 14.5 \%)$ compared to pre-intervention data. No changes $(p>0.05)$ in mean concentric VM $(3.7 \pm 8.0 \%$ to $12.7 \pm 8.6 \%)$ or Glut $(0.3 \pm 10.4 \%$ to $7.0 \pm 7.5 \%)$ EMG or peak concentric VL $(0.6 \pm 5.8 \%$ to $4.5 \pm 4.7 \%), \operatorname{VM}(0.3 \pm 5.1 \%$ to $9.2 \pm 4.1 \%)$ or Glut $(0.2 \pm 9.2 \%$ to $7.1 \pm 7.7 \%$ ) EMG were observed at any time point (see Table 4).

Significant $(p<0.05)$ correlations were observed between the change in CMJ height (preintervention to $8 \mathrm{~min}$ post-intervention, i.e. where the greatest mean increase in jump height occurred) and changes in peak power $(\mathrm{r}=0.82)$ during VR. No significant correlations $(p>$ 0.05) were found between change in CMJ height and changes in peak normalized RFD ( $\mathrm{r}=$ 0.27), peak knee angular velocity $(r=-0.21)$, mean concentric VL EMG $(r=0.17)$ or peak eccentric kinetic energy $(r=0.32)$.

Table 4 about here in countermovement vertical jump $(\mathrm{CMJ})$ performance after traditional free-weight $(\mathrm{FWR})$ and variable (VR) resistance squat exercises were performed following a comprehensive taskspecific, warm-up routine. The first hypothesis can be partially accepted as the lack of change in any measure following the FWR condition suggests that no additional benefit (i.e. 
423 PAP/PAPE effect) was derived from the inclusion of intense loading from FWR exercise (i.e.

424 the conditioning activity), contrary to the improvement in jump height following the use of

425 elastic bands. This finding contrasts those of previous studies where the performance of heavy 426 squat lifts increased CMJ height, ${ }^{21,32}$ and other literature reporting an increase in tasks 427 including sprint running performance. ${ }^{33}$ However, those previous studies either did not report 428 the use of other warm-up activities or only included a light cardiovascular warm-up rather than 429 a more comprehensive task-specific warm-up including progressively intense task-specific 430 muscular contractions. The current finding of a lack of effect of a back squat conditioning 431 activity after a comprehensive task-specific warm-up (see Figure 2) is, however, consistent 432 with a previous report of an absence of change in vertical jump performance when dynamic warm-up exercises were employed prior to a set of back squats. ${ }^{17}$ These data are indicative 434 that a lack of a comprehensive task-specific warm-up may enable further augmentation of 435 performance after squats were performed, but may be of limited relevance to athletes, strength 436 trainers and recreational exercisers who would customarily perform a thorough warm-up. That 437 is, the high-intensity conditioning activities might only increase performance when the warm438 up would otherwise be insufficient to promote maximal performance. Collectively, these 439 findings indicate that the previously reported 'potentiating' effects of heavy free-weight back 440 squat exercise on subsequent CMJ performance ${ }^{16,21}$ may be a consequence of study design, 441 where the limited use of warm-up protocols provided an opportunity for further performance 442 augmentation after the baseline tests. Furthermore, inconsistencies in PAP responses ${ }^{12,16,17}$ 443 may depend on fatigue-potentiation or perseveration-potentiation interactions and their 444 influence on subsequent performance therefore new strategies for designing warm-up protocols 445 and optimal recovery periods following conditioning contractions are vital in order to induce a 446 potentiation effect. 
448 Despite FWR squat lifts having no effect on CMJ performance, a significant increase in jump 449 height was achieved following the VR conditioning activity at all time points $(30 \mathrm{~s}, 4 \mathrm{~min}, 8$ 450 min and 12 min; see Figure 2), which suggests a prolonged 'potentiating' effect was evoked, 451 i.e. post-activation performance enhancement; PAPE. ${ }^{9}$ Thus, the second experimental 452 hypothesis, that jump height would be increased following the VR intervention, can be 453 accepted. These data are consistent with a previous study ${ }^{34}$ in which box squats incorporating 454 elastic band resistance acutely increased power output during subsequent CMJ tasks. However, 455 in the present study it was shown that this effect can be evoked even after completion of a 456 comprehensive task-specific warm-up, which was not included in previous studies. Although 457 each maximal CMJ may possibly potentiate the next one, no significant improvement occurred 458 in the FWR condition, therefore these jumps were unlikely to explain the increased 459 performance in VR. Previous studies showed that only seconds or a few minutes are needed 460 to recover from a short bout of maximal-effort exercise (e.g. less than $1 \mathrm{~min}$ for recovery from 461 a maximal squat ${ }^{35}$ or bench press lifts $\left.^{36}\right)$, thus it is unlikely that fatigue is a factor influencing 462 the findings of the present study as a significant increase was observed across all time points. 463 The use of elastic bands reduces the effective load near the "sticking point" in the early 464 concentric phase of the squat lift but then allows for greater loading later in the lift as the 465 effective mechanical advantage is increased. ${ }^{2}$ The ability for muscles to operate closer to their 466 maximum force capacity through a greater proportion of the lift may therefore enhance 467 subsequent muscle force output and elicit a greater dynamic muscle performance (i.e. increase 468 in CMJ height), even when a comprehensive task-specific warm-up is already completed. 469 Collectively, these data indicate that the use of elastic bands, which alter the loading strategy 470 during the lift, provides a more effective warm-up than either warm-up alone or warm-up that 471 also includes traditional free-weight resistance exercises. 
473 In the present study significant changes in force production (peak power and RFD; see Table

474 2) at all time points in the VR warm-up condition were consistent with the changes in jump 475 height. However, peak hip, knee and ankle, flexion angle, peak eccentric kinetic energy, the 476 impulse- and time-based asymmetry indexes remained unchanged and no change was detected 477 in $\mathrm{K}_{\mathrm{vert}}$ (see Tables 2 and 3), which is consistent with previous research. ${ }^{30}$ Accordingly, 478 changes in jump kinematics cannot explain the changes in force production or jump height. 479 The third hypothesis, that both kinetic and kinematic parameters would be altered by elastic 480 band-resisted squat lifts, can therefore only be partially accepted. The changes in peak jump 481 power were significantly correlated with the changes in CMJ height, however a poor 482 relationship was identified between changes in RFD and CMJ height. This latter finding is 483 consistent with a previous report ${ }^{37}$ in which stretch-shortening cycle test performances were not statistically related to RFD measured during the test. The poor relationship may be partly explained by the participants being well strength-trained yet relatively untrained in explosive power-based exercises, and thus unable to rapidly reach peak force. ${ }^{38}$ However, further research on power-trained athletes is needed to fully elucidate the importance of training status.

489 A number of mechanisms relating to stretch-shorten cycle efficiency may have contributed to the increased jump height, including a more rapid muscle stretch resulting from force potentiation, ${ }^{3939}$ greater elastic energy storage in the muscle, ${ }^{40}$ an increased time of muscle 492 activation, ${ }^{40,41}$ an augmented pre-load effect, ${ }^{42}$ force and stiffness augmentation from stretch 493 reflexes, ${ }^{41}$ or changes in relative contributions of muscle and tendon allowing the muscle to 494 operate at lower shortening speeds and over shorter distances. ${ }^{43}$ Whilst it is difficult to assess 495 the effects of each, the peak eccentric kinetic energy and both impulse- and time-based 496 asymmetry indexes remained unchanged after VR, indicating that the total energy available for 497 storage in elastic structures (eccentric kinetic energy), the kinematic pattern adopted to make 
498

499

500

501

502

503

504

505

506

507

508

509

510

511

512

513

514

515

516

517

518

519

520

521

522

use of it (asymmetry indexes), ${ }^{29}$ and the time for force application and likely contribution of stretch reflexes, were also unchanged. Nonetheless, increases in peak power and concentric knee angular velocity were observed.

Nonetheless, a more plausible explanation for the increase in force production, and thus jump height, may be found in the increased knee extensor muscle activity detected in the concentric phase (VL EMG increased 27.5-33.4\% across time points; see Table 4). Thus, the fourth hypothesis, that extensor muscle activity would be increased, can be accepted. The greater increase in EMG activity in VL than VM or Glut is consistent with previous reports of greater VL EMG in the concentric phase of a CMJ after both low- and moderate-intensity squat warmups $^{32}$ and would likely have resulted from an increased motor unit firing frequency. ${ }^{44}$ In fact, Nikolaidou et al. ${ }^{45}$ found that a greater jump height was achieved during CMJ compared to squat jump which was consistent with an increased VL activation during the push off phase. Increased phosphorylation of the myosin light chain leading to an increase in myofilament $\mathrm{Ca}^{2+}$ sensitivity and force output (i.e. classic PAP) may have contributed to the increase in CMJ, although it resolves completely within about $5 \min ^{11}$ thus its effect at $4-12 \min$ would have been negligible. Although other mechanisms such as increases in muscle temperature ${ }^{5}$ (not examined in the present study) may have contributed to the increase in jump height it remains likely that the change in muscle activation was the major factor influencing the improvement in CMJ performance. The increased muscle activity and consequent increase in peak power output in the concentric phase would have allowed a greater jump height without changes in kinematics or stretch-shorten cycle efficiency (i.e. without changes in eccentric knee angular velocity, eccentric kinetic energy, impulse- and time-based asymmetry indexes or $\left.\mathrm{K}_{\mathrm{vert}}\right)$. The most likely explanation for the finding is that the variation in muscle force requirements imposed by the use of elastic band resistance influenced muscle recruitment patterns and 
523 ultimately increased concentric force output. ${ }^{19}$ The current findings hint at the possibility that

524 manipulation of loading strategies during warm-up exercises might beneficially alter muscle

525 recruitment amplitude or timing and result in greater performances than achieved through

526 traditional high-intensity, task-specific warm-ups alone. This hypothesis should be explicitly

527 examined in future studies. It is important to note that it was not possible to measure muscle

528 temperatures in the current study. However, muscle temperature would likely have increased

529 substantially during the comprehensive task-specific warm-up so temperature may have

530 remained constant (i.e. in an optimum zone) for a longer time, and any further small increase

531 in temperature from the conditioning activities would have been similar between conditions.

532 This may have allowed the improved muscle activation to result in a greater jump performance

533 and for the increased activation to persist for a longer time. Thus, although it remains to be

534 explicitly examined in future, it can be considered unlikely that muscle temperature differences

535 could explain the between-condition differences in jump performance.

536

537 PERSPECTIVE

538 The completion of brief, high-load free-weight squat exercise following a comprehensive task-

539 specific warm-up failed to alter CMJ height, force/power production or movement pattern.

540 These findings are suggestive that the previously-observed 'potentiating' effect of squat

541 exercise may be a consequence of limited warm-up. The beneficial effect of a free-weight squat

542 strategy to potentiate the system may therefore, be minimal in athletic populations that typically

543 perform high-intensity, task-specific warm-up routines prior to maximal exercise tasks.

544 However, the use of elastic band resistance during these squats resulted in significant increases

545 in jump height, peak power, peak concentric knee angular velocity and peak RFD, as well as

546 increased VL EMG activity in the concentric (propulsive) phase of the jump, which did not

547 return to baseline after $12 \mathrm{~min}$ despite a comprehensive task-specific warm-up being 
548 completed. The results suggest that the inclusion of tasks in which force-time parameters differ

549 from the outcome task (CMJ in the current study) might evoke positive acute adaptations in

550 addition to those achieved through warm-up alone. Further research is required to determine

551 whether similar effects are observed following different warm-up strategies and in different

552 athletic tasks, as well as in other populations.

553

554 ACKNOWLEDGEMENTS

555 No funding was received for this work. No conflicts of interest exist.

556

557

558

559

560

561

562

563

564

565

566

567

568

569

570

571

572 


\section{REFERENCES}

574 1. Tillin NA, Bishop D. Factors modulating post-activation potentiation and its effect on 575 performance on subsequent explosive activities. Sports Med 2009;39:147-166.

576 2. Mina MA, Blazevich AJ, Giakas G, Kay AD. The influence of variable resistance loading 577 on subsequent free-weight maximal back squat performance. J Strength Cond Res $578 \quad 2014 ; 28: 2988-2996$.

579 3. Woods K, Bishop P, Jones E. Warm-up and stretching in the prevention of muscular injury. $580 \quad$ Sports Med 2007;37:1089-1099.

581 4. Hodgson M, Docherty D, Robbins D. Post activation potentiation: Underlying physiology 582 and implication for motor performance. Sports Med 2005;35:585-595.

583 5. Racinais S, Oksa J. Temperature and neuromuscular function. Scand J Med Sci Sports 584 2010;20:11-18.

6. Moore RL, Stull JT. Myosin light chain phosphorylation in fast and slow skeletal muscles in situ. Am J Physiol Cell Physiol 1984;247:462-471.

7. Trimble MH, Harp SS. Postexercise potentiation of the H-reflex in human. Med Sci Sports Exerc 1998;30:933-941.

8. Hamada T, Sale DG, MacDougall JD, Tarnopolsky MA. Postactivation potentiation, fibre type and twitch contraction time in human knee extensor muscles. J Appl Physiol $2000 ; 88: 2131-2137$.

9. Cuenca-Fernandez F, Smith IC, Jordan MJ, et al. Nonlocalized postactivation performance enhancement (PAPE) effects in trained athletes: a pilot study. Appl Physiol Nutr Metab $2017 ; 42: 1122-1125$.

10. Jo E, Judelson DA, Brown LE, Coburn JW, Dabbs NC. Influence of recovery duration after a potentiating stimulus on muscular power in recreationally trained individuals. $\mathrm{J}$ Strength Cond Res 2010;24:343-347. 
598 11. MacIntosh BR, Robillard ME, Tomaras EK. Should postactivation potentiation be the goal 599 of your warm-up? Appl Physiol Nutr Metab 2012;37:546-550.

600 12. Young WB, Jenner A, Griffiths K. Acute enhancement of power performance from heavy $601 \quad$ load squats. J Strength Cond Res 1998;12:82-84.

602 13. Arabatzi F, Patikas D, Zafeiridis A, et al. The post-activation potentiation effect on squat 603 jump performance: Age and sex effect. Pediatr Exerc Sci 2014;26:187-194.

604 14. Baker DG. Increase in jump squat peak external power output when combined with 605 accommodating resistance box squats during contrasting resistance complex training with 606 short rest periods. J Aust Strength Cond 2008;6:10-18.

15. Wilson JM, Duncan NM, Marin PJ, et al. Meta-analysis of post activation potentiation and power: Effect of conditioning activity, volume, gender, rest periods, and training status. J Strength Cond Res 2013;27:854-859.

16. Gourgoulis V, Aggeloussis N, Kasimatis P, Mavromatis G, Garas A. Effect of a submaximal half-squats warm-up program on vertical jumping ability. J Strength Cond Res $2003 ; 17: 342-344$.

17. Witmer CA, Davis SE, Moir GL. The acute effects of back squats on vertical jump performance in men and women. J Sports Sci Med 2010;9:206-213.

18. Elliott BC, Wilson GJ. A biomechanical analysis of the sticking region in the bench press. Med Sci Sports Exerc 1989;21:450-462.

19. Israetel MA, McBride JM, Nuzzo JL, Skinner JD, Dayne AM. Kinetic and kinematic difference between squat performed with and without elastic bands. J Strength Cond Res 2010;24:190-194.

20. Wallace BJ, Winchester JB, McGuigan MR. Effects of elastic bands on force and power characteristics during the back squat exercise. J Strength Cond Res 2006;20:268-272. 
622 21. Lowery RP, Duncan NM, Loenneke JP, et al. The effects of potentiating stimuli intensity 623 under varying rest periods on vertical jump performance and power. J Strength Cond Res $624 \quad 2012 ; 26: 3320-3325$.

625 22. Mina MA, Blazevich AJ, Giakas G, Seitz LB, Kay AD. Chain-loaded variable resistance 626 warm-up improves free-weight maximal back squat performance. Eur J Sport Sci $627 \quad 2016 ; 16: 932-939$.

628 23. Golas A, Wilk M, Stastny P, Maszczyk A, Pajerska K, Zajac A. Optimizing half squat 629 postactivation potential load in squat jump training for eliciting relative maximal power in $630 \quad$ ski jumpers. J Strength Cond Res 2017;31:3010-3017.

631 24. Sheppard JM, Triplett NT. Program design for resistance training. In: Haff GG, Triplett 632 NT, ed. Essentials of strength training and conditioning. $4^{\text {th }}$ ed. Champaign: Human $633 \quad$ Kinetics; 2016. 452-456 p.

25. Kilduff LP, Bevan HR, Kingsley MIC, et al. Post activation potentiation in professional rugby players: Optimal recovery. J Strength Cond Res 2007;21:1134-1138.

26. Woltring HJ. A fortan package for generalized cross-validatory spline smoothing and differentiation. Adv Eng Softw 1986;8:104-113.

27. Davis III RB, Ounpuu S, Tyburski D, Gage JR. A gait analysis data collection and reduction technique. Hum Mov Sci 1991;10:575-587.

28. Kibele A. Possibilities and limitations in biomechanical analysis of countermovement jumps: a methodological study. J Appl Biomech 1998;14:105-117.

29. Cavagna GA. Storage and utilization of elastic energy in skeletal muscle. Exerc Sport Sci 643 Rev 1977;5:89-129.

30. Boullosa DA, Abreu L, Beltrame LG, Behm DG. The acute effect of different half squat set configurations on jump potentiation. J Strength Cond Res 2013;27:2059-2066.

31. Cavagna GA. Force platforms as ergometers. J Appl Physiol 1985;39:174-179. 
647 32. Sotiropoulos K, Smilios I, Christou M, et al. Effect of warm-up on vertical jump 648 performance and muscle electrical activity using half-squats and moderate intensity. J $649 \quad$ Sports Sci Med 2010;9:326-331.

650 33. Chatzopoulos DE, Michailidis CJ, Giannakos AK, et al. Postactivation potentiation effects 651 after heavy resistance exercise on running speed. J Strength Cond Res 2007;21:1278$652 \quad 1281$.

653 34. Buttifant D, Hrysomallis C. Effect of various practical warm-up protocols on acute lower654 body power. J Strength Cond Res 2015;29:656-660.

655 35. Hitchcock HC. Recovery of short-term power after dynamic exercise. J Appl Physiol $656 \quad 1987 ; 67: 677-681$.

657 36. Matuszak ME, Fry AC, Weiss LW, Ireland TR, McKnight MM. Effect of rest interval 658 length on repeated 1 repetition maximum back squat. J Strength Cond Res 2003;17:634659637.

660 37. Wilson G, Lyttle A, Ostrowski K, Murphy A. Assessing dynamic performance: A 661 comparison of rate of force development tests. J Strength Cond Res 1995;9:176-181.

662 38. Moir G, Sanders R, Button C, Glaister M. The influence of familiarization on the reliability 663 of force variables measured during unloaded and loaded vertical jumps. J Strength Cond 664 Res 2005;19:140-145.

39. Rassier DE, Herzog W. Considerations on the history dependence on muscle contraction. J Appl Physiol 2004;96:419-427.

40. Finni T, Ikegawa S, Lepola V, Komi PV. Comparison of force-velocity relationships of vastus lateralis muscle in isokinetic and in stretch-shortening cycle exercises. Acta Physiol Scan 2003;177:483-491.

41. Bobbert MF, Gerritsen KGM, Litjens MCA, van Soest AJ. Why is countermovement jump height greater than squat jump height? Med Sci Sports Exerc 1996;28:1402-1412. 
672 42. Finni T, Ikegawa S, Komi PV. Concentric force enhancement during human movement. $673 \quad$ Acta Physiol Scan 2001;173:369-377.

674 43. Hill AV. The heat of shortening and the dynamic constants of the muscle. Proc R Soc B 675 Biol Sci 1938;843:136-195.

676 44. Heckman CJ, Enoka RM. Motor Unit. Compr Physiol 2012;2:2629-2682.

677 45. Nikolaidou ME, Marzilger SB, Mersmann F, Arampatzis A. Operating length and velocity

678 of human M. vastus lateralis fascicles during vertical jumping. R Soc Open Sci $679 \quad 2017 ; 4: 170185$.

680

681

682

683

684

685

686

687

688

689

690

691

692 


\section{TABLE AND FIGURE LEGENDS}

694 Table 1. Study design timeline. Acronyms: CMJ = countermovement vertical jump; FWR = 695 free-weight resistance; VR = variable resistance.

696

697

Table 2. Kinetic measures of vertical jump performance across all time points following the 698 free-weight resistance and elastic band warm-up conditions (values are reported as mean $\pm \mathrm{SE}$; $699 * p<0.05$ compared to pre-intervention and FWR condition). Acronyms: Pre $=$ pre700 intervention; $\mathrm{FWR}=$ free-weight resistance; $\mathrm{VR}=$ variable resistance; $\mathrm{K}_{\mathrm{vert}}=$ vertical stiffness; $701 \mathrm{RFD}=$ rate of force development.

702

703

Table 3. Kinematic measures of vertical jump performance across all time points following 704 the free-weight resistance and elastic band warm-up conditions (values are reported as mean $\pm \mathrm{SE} ;{ }^{*} p<0.05$ compared to pre-intervention). Acronyms: Pre $=$ pre-intervention; FWR $=$

706 free-weight resistance; $\mathrm{VR}=$ variable resistance; $\mathrm{ECC}=$ eccentric $; \mathrm{CON}=$ concentric.

Table 4. Normalized mean and peak VL, VM and Glut EMG amplitudes measured during vertical jumps across all time points following free-weight resistance and elastic band warmup squat conditions (values are reported as mean $\pm \mathrm{SE} ;{ }^{*} p<0.05$ compared to pre-intervention and FWR condition). Acronyms: $\mathrm{VL}=$ vastus lateralis; $\mathrm{VM}=$ vastus medialis; Glut = gluteus maximum; $\mathrm{EMG}=$ electromyogram $; \mathrm{MVC}=$ maximum voluntary contraction; $\mathrm{FWR}=$ freeweight resistance; $\mathrm{VR}=$ variable resistance; $\mathrm{ECC}=$ eccentric; $\mathrm{CON}=$ concentric. 
715 Figure 1. Exemplar data from a subject depicting CMJ height, ground reaction force, knee 716 angular velocity, knee flexion angle and VL EMG activity at $8 \mathrm{~min}$ following the free-weight 717 resistance and elastic band warm-up squat conditions. VL = vastus lateralis; $\mathrm{CMJ}=$ 718 countermovement vertical jump; FWR = free-weight resistance; VR = variable resistance.

719

720 Figure 2. Mean countermovement vertical jump height following free-weight resistance and 721 elastic band warm-up squat conditions. *Significant increases $(5.3-6.5 \% ; \mathrm{p}<0.05)$ in vertical 722 jump performance were achieved across all time points following the VR warm-up condition 723 compared to pre-intervention and the FWR warm-up condition. Pre = pre-intervention; FWR $724=$ free-weight resistance; $\mathrm{VR}=$ variable resistance. 
Table 1. Study design timeline.

\begin{tabular}{lc}
\hline Task & Time (min) \\
\hline 5 -min cycle & $0-5.0$ \\
5 unloaded squats $(1 \mathrm{~s} / 1 \mathrm{~s})$ & $5.0-6.0$ \\
5 unloaded squats $(2 \mathrm{~s} / 2 \mathrm{~s})$ & $6.0-7.0$ \\
5 CMJs $(70 \%)$ & $7.5-8.5$ \\
Single CMJs every $30 \mathrm{~s}(100 \%)$ & $9.0-11.0$ \\
CMJ Test 1 & $13.0-13-5$ \\
FWR or VR squats & $14.5-15.0$ \\
CMJ Tests $(2-5)$ & $15.5,19.5,23.5,27.5$
\end{tabular}

Acronyms: $\mathrm{CMJ}=$ countermovement vertical jump; FWR = free-weight resistance; VR = variable resistance. 
Table 2. Kinetic measures of vertical jump performance across all time points following the free-weight and variable resistance warm-up conditions (values are reported as mean $\pm \mathrm{SE} ;{ }^{*} p<0.05$ compared to pre-intervention and FWR condition).

\begin{tabular}{|c|c|c|c|c|c|c|}
\hline Measure & Condition & Pre & $30 \mathrm{~s}$ & $4 \mathrm{~min}$ & $8 \mathrm{~min}$ & $12 \mathrm{~min}$ \\
\hline \multirow[t]{2}{*}{ Peak Power (W) } & FWR & $49.3 \pm 1.9$ & $50.3 \pm 1.5$ & $50.8 \pm 1.7$ & $49.2 \pm 2.2$ & $50.3 \pm 1.7$ \\
\hline & VR & $49.0 \pm 1.7$ & $51.3 \pm 1.8^{*}$ & $51.8 \pm 1.6^{*}$ & $51.0 \pm 1.7^{*}$ & $51.2 \pm 1.7^{*}$ \\
\hline \multirow{2}{*}{$\begin{array}{l}\text { Peak Eccentric Kinetic } \\
\text { Energy }(\mathrm{J})\end{array}$} & FWR & $87.4 \pm 7.7$ & $95.8 \pm 9.0$ & $90.8 \pm 9.4$ & $90.3 \pm 7.7$ & $88.0 \pm 9.0$ \\
\hline & VR & $94.3 \pm 8.0$ & $93.6 \pm 7.3$ & $87.9 \pm 7.2$ & $88.8 \pm 6.9$ & $82.4 \pm 5.9$ \\
\hline \multirow{2}{*}{$\begin{array}{l}\text { Impulse asymmetry index } \\
(\mathrm{N} \cdot \mathrm{s})\end{array}$} & FWR & $2.9 \pm 0.1$ & $2.8 \pm 0.1$ & $2.9 \pm 0.1$ & $2.9 \pm 0.1$ & $2.9 \pm 0.1$ \\
\hline & VR & $2.8 \pm 0.1$ & $2.9 \pm 0.1$ & $2.9 \pm 0.1$ & $2.9 \pm 0.1$ & $2.9 \pm 0.1$ \\
\hline \multirow[t]{2}{*}{ Time asymmetry index (ms) } & FWR & $1.5 \pm 0.1$ & $1.4 \pm 0.2$ & $1.5 \pm 0.2$ & $1.3 \pm 0.2$ & $1.2 \pm 0.2$ \\
\hline & VR & $1.4 \pm 0.2$ & $1.5 \pm 0.2$ & $1.1 \pm 0.2$ & $1.4 \pm 0.2$ & $1.2 \pm 0.2$ \\
\hline \multirow[t]{2}{*}{$\mathrm{K}_{\mathrm{vert}}\left(\mathrm{N} \cdot \mathrm{m}^{-1} \cdot \mathrm{kg}^{-1}\right)$} & FWR & $70.8 \pm 6.0$ & $72.6 \pm 3.8$ & $70.5 \pm 3.6$ & $69.0 \pm 3.7$ & $72.0 \pm 3.8$ \\
\hline & VR & $69.9 \pm 5.0$ & $73.3 \pm 3.9$ & $73.5 \pm 4.5$ & $74.6 \pm 3.7$ & $74.4 \pm 4.5$ \\
\hline \multirow{2}{*}{$\begin{array}{l}\text { Peak normalized RFD } \\
\left(\mathrm{N} \cdot \mathrm{s}^{-1}\right)\end{array}$} & FWR & $134.2 \pm 11.3$ & $147.1 \pm 12.2$ & $132.5 \pm 10.8$ & $141.8 \pm 13.5$ & $118.2 \pm 7.5$ \\
\hline & VR & $126.1 \pm 6.7$ & $149.8 \pm 12.8^{*}$ & $143.2 \pm 11.7^{*}$ & $149.2 \pm 9.0^{*}$ & $147.7 \pm 13.7^{*}$ \\
\hline
\end{tabular}

Acronyms: Pre = pre-intervention; $\mathrm{FWR}=$ free-weight resistance; $\mathrm{VR}=$ variable resistance; $\mathrm{K}_{\mathrm{vert}}=$ vertical stiffness; RFD = rate of force development. 
Table 3. Kinematic measures of vertical jump performance across all time points following the free-weight and variable resistance warm-up conditions (values are reported as mean $\pm \mathrm{SE} ; * p<0.05$ compared to preintervention).

\begin{tabular}{|c|c|c|c|c|c|c|c|}
\hline Measure & Mode & Condition & Pre & $30 \mathrm{~s}$ & $4 \min$ & $8 \min$ & $12 \mathrm{~min}$ \\
\hline \multirow{4}{*}{$\begin{array}{l}\text { Peak hip angular } \\
\text { velocity }\left({ }^{\circ} \cdot \mathrm{s}^{-1}\right)\end{array}$} & ECC & FWR & $301.1 \pm 9.5$ & $302.2 \pm 10.0$ & $294.4 \pm 9.8$ & $291.4 \pm 6.9$ & $292.8 \pm 9.2$ \\
\hline & & VR & $298.2 \pm 7.1$ & $305.2 \pm 8.3$ & $300.2 \pm 8.3$ & $302.6 \pm 8.8$ & $297.3 \pm 8.7$ \\
\hline & $\mathrm{CON}$ & FWR & $584.6 \pm 15.6$ & $605.4 \pm 18.8$ & $591.9 \pm 20.2$ & $575.7 \pm 20.7$ & $576.2 \pm 15.8$ \\
\hline & & $\overline{V R}$ & $572.2 \pm 17.1$ & $591.9 \pm 20.2$ & $593.0 \pm 22.1$ & $588.2 \pm 19.3$ & $580.8 \pm 21.7$ \\
\hline \multirow{4}{*}{$\begin{array}{l}\text { Peak knee angular } \\
\text { velocity }\left({ }^{\circ} \cdot \mathrm{s}^{-1}\right)\end{array}$} & ECC & FWR & $343.2 \pm 13.6$ & $341.0 \pm 11.4$ & $332.6 \pm 11.9$ & $343.8 \pm 13.7$ & $340.5 \pm 14.3$ \\
\hline & & VR & $352.1 \pm 14.3$ & $364.9 \pm 15.7$ & $353.5 \pm 13.9$ & $363.0 \pm 15.0$ & $347.5 \pm 16.6$ \\
\hline & $\mathrm{CON}$ & FWR & $956.4 \pm 23.6$ & $971.6 \pm 24.6$ & $969.3 \pm 26.7$ & $939.6 \pm 27.9$ & $959.6 \pm 25.1$ \\
\hline & & VR & $937.0 \pm 23.8$ & $966.0 \pm 28.8^{*}$ & $975.7 \pm 29.7^{*}$ & $966.9 \pm 26.2^{*}$ & $964.2 \pm 24.5^{*}$ \\
\hline \multirow{4}{*}{$\begin{array}{l}\text { Peak ankle angular } \\
\text { velocity }\left({ }^{\circ} \cdot \mathrm{s}^{-1}\right)\end{array}$} & ECC & FWR & $108.1 \pm 10.0$ & $117.6 \pm 12.0$ & $109.7 \pm 12.2$ & $112.0 \pm 10.9$ & $114.4 \pm 10.8$ \\
\hline & & VR & $121.1 \pm 12.8$ & $118.7 \pm 9.8$ & $120.3 \pm 9.0$ & $112.8 \pm 7.1$ & $104.5 \pm 5.0$ \\
\hline & $\mathrm{CON}$ & FWR & $745.4 \pm 23.4$ & $733.7 \pm 25.5$ & $728.2 \pm 18.9$ & $707.9 \pm 25.3$ & $721.5 \pm 27.0$ \\
\hline & & VR & $717.9 \pm 21.3$ & $723.6 \pm 22.2$ & $731.7 \pm 23.6$ & $735.1 \pm 28.1$ & $739.4 \pm 19.1$ \\
\hline \multirow{2}{*}{$\begin{array}{l}\text { Peak hip flexion } \\
\text { angle }\left(^{\circ}\right)\end{array}$} & & FWR & $79.3 \pm 2.0$ & $82.7 \pm 2.1$ & $81.8 \pm 1.7$ & $82.1 \pm 2.4$ & $81.8 \pm 2.2$ \\
\hline & & VR & $81.5 \pm 1.9$ & $83.2 \pm 1.4$ & $83.3 \pm 1.9$ & $83.4 \pm 1.5$ & $82.8 \pm 1.5$ \\
\hline \multirow{2}{*}{$\begin{array}{l}\text { Peak knee flexion } \\
\text { angle }\left({ }^{\circ}\right)\end{array}$} & & FWR & $71.7 \pm 2.9$ & $73.3 \pm 3.0$ & $71.1 \pm 2.8$ & $72.0 \pm 2.7$ & $71.9 \pm 3.3$ \\
\hline & & VR & $71.8 \pm 3.5$ & $72.6 \pm 3.4$ & $74.2 \pm 3.3$ & $75.2 \pm 2.8$ & $75.4 \pm 3.5$ \\
\hline \multirow{2}{*}{$\begin{array}{l}\text { Peak ankle flexion } \\
\text { angle }\left({ }^{\circ}\right)\end{array}$} & & FWR & $32.7 \pm 1.6$ & $32.6 \pm 1.4$ & $32.7 \pm 1.4$ & $33.0 \pm 1.4$ & $33.2 \pm 1.4$ \\
\hline & & VR & $33.8 \pm 1.6$ & $34.7 \pm 1.6$ & $34.8 \pm 1.6$ & $35.2 \pm 1.6$ & $34.8 \pm 1.8$ \\
\hline
\end{tabular}

Acronyms: Pre $=$ pre-intervention; $\mathrm{FWR}=$ free-weight resistance; $\mathrm{VR}=$ variable resistance; $\mathrm{ECC}=$ eccentric; $\mathrm{CON}=$ concentric. 
Table 4. Normalized mean and peak VL, VM and Glut EMG amplitudes measured during vertical jumps across all time points following free-weight and variable resistance warm-up squat conditions (values are reported as mean $\pm \mathrm{SE} ;{ }^{*} p<0.05$ compared to pre-intervention and FWR condition).

\begin{tabular}{|c|c|c|c|c|c|c|c|}
\hline Measure & Mode & Condition & Pre & $30 \mathrm{~s}$ & $4 \mathrm{~min}$ & $8 \mathrm{~min}$ & $12 \mathrm{~min}$ \\
\hline \multirow{4}{*}{$\begin{array}{l}\text { Mean VL EMG } \\
(\% \text { MVC) }\end{array}$} & $\mathrm{ECC}$ & FWR & $31.9 \pm 2.1$ & $31.5 \pm 3.4$ & $30.5 \pm 3.0$ & $30.0 \pm 2.0$ & $29.5 \pm 2.9$ \\
\hline & & VR & $28.5 \pm 2.1$ & $30.2 \pm 2.2$ & $31.9 \pm 2.3$ & $31.5 \pm 1.8$ & $32.0 \pm 2.2$ \\
\hline & $\mathrm{CON}$ & FWR & $82.4 \pm 6.1$ & $83.9 \pm 6.4$ & $78.4 \pm 8.5$ & $72.5 \pm 4.6$ & $73.4 \pm 6.0$ \\
\hline & & VR & $85.4 \pm 7.8$ & $108.2 \pm 13.3^{*}$ & $110.2 \pm 12.2^{*}$ & $107.5 \pm 10.5^{*}$ & $102.6 \pm 9.4^{*}$ \\
\hline \multirow{4}{*}{$\begin{array}{l}\text { Peak VL EMG } \\
(\% \text { MVC) }\end{array}$} & ECC & FWR & $89.2 \pm 6.7$ & $94.7 \pm 8.0$ & $90.4 \pm 6.9$ & $85.0 \pm 6.7$ & $84.0 \pm 6.0$ \\
\hline & & VR & $91.6 \pm 4.9$ & $95.0 \pm 8.0$ & $94.2 \pm 6.1$ & $90.7 \pm 4.7$ & $90.6 \pm 5.9$ \\
\hline & $\mathrm{CON}$ & FWR & $112.4 \pm 8.0$ & $123.7 \pm 9.6$ & $116.9 \pm 7.1$ & $112.8 \pm 6.7$ & $112.2 \pm 6.3$ \\
\hline & & VR & $114.3 \pm 5.8$ & $115.8 \pm 10.2$ & $117.4 \pm 7.6$ & $111.2 \pm 6.6$ & $108.1 \pm 6.6$ \\
\hline \multirow{4}{*}{$\begin{array}{l}\text { Mean VM EMG } \\
(\% \mathrm{MVC})\end{array}$} & ECC & FWR & $36.3 \pm 2.7$ & $33.9 \pm 4.1$ & $32.6 \pm 3.3$ & $32.2 \pm 2.6$ & $33.0 \pm 3.4$ \\
\hline & & VR & $37.9 \pm 3.8$ & $40.2 \pm 3.8$ & $38.1 \pm 3.3$ & $39.9 \pm 2.9$ & $38.1 \pm 3.3$ \\
\hline & $\mathrm{CON}$ & FWR & $94.9 \pm 5.0$ & $95.3 \pm 10.9$ & $85.1 \pm 8.4$ & $85.1 \pm 6.4$ & $87.7 \pm 7.3$ \\
\hline & & VR & $90.2 \pm 9.2$ & $96.0 \pm 7.9$ & $94.7 \pm 7.5$ & $88.8 \pm 5.9$ & $87.2 \pm 6.2$ \\
\hline \multirow{4}{*}{$\begin{array}{l}\text { Peak VM EMG } \\
(\% M V C)\end{array}$} & ECC & FWR & $98.6 \pm 7.9$ & $96.9 \pm 7.4$ & $92.9 \pm 6.7$ & $88.0 \pm 6.1$ & $89.0 \pm 6.1$ \\
\hline & & VR & $111.3 \pm 9.5$ & $114.4 \pm 11.2$ & $108.1 \pm 10.2$ & $104.7 \pm 8.1$ & $97.3 \pm 6.7$ \\
\hline & $\mathrm{CON}$ & FWR & $132.0 \pm 12.2$ & $128.7 \pm 11.5$ & $120.5 \pm 9.5$ & $118.2 \pm 8.6$ & $118.8 \pm 9.1$ \\
\hline & & VR & $150.6 \pm 12.8$ & $149.0 \pm 14.4$ & $143.4 \pm 12.5$ & $140.8 \pm 11.8$ & $127.7 \pm 8.9$ \\
\hline \multirow{4}{*}{$\begin{array}{l}\text { Mean Glut EMG } \\
(\% \mathrm{MVC})\end{array}$} & $\mathrm{ECC}$ & FWR & $20.0 \pm 1.9$ & $22.4 \pm 2.8$ & $21.3 \pm 2.3$ & $19.7 \pm 1.7$ & $21.2 \pm 1.9$ \\
\hline & & VR & $21.7 \pm 2.5$ & $23.2 \pm 2.6$ & $22.5 \pm 2.4$ & $22.5 \pm 2.2$ & $21.7 \pm 2.0$ \\
\hline & $\mathrm{CON}$ & FWR & $81.1 \pm 10.2$ & $84.4 \pm 14.8$ & $83.9 \pm 12.1$ & $78.6 \pm 8.7$ & $75.1 \pm 9.2$ \\
\hline & & VR & $84.4 \pm 14.0$ & $87.1 \pm 14.2$ & $78.3 \pm 10.1$ & $77.4 \pm 8.5$ & $76.8 \pm 9.8$ \\
\hline \multirow{4}{*}{$\begin{array}{l}\text { Peak Glut EMG } \\
(\% \mathrm{MVC})\end{array}$} & $\mathrm{ECC}$ & FWR & $72.6 \pm 7.0$ & $78.3 \pm 7.5$ & $76.4 \pm 7.2$ & $74.6 \pm 7.1$ & $71.7 \pm 6.9$ \\
\hline & & VR & $79.1 \pm 9.4$ & $82.0 \pm 9.5$ & $76.7 \pm 8.9$ & $74.7 \pm 5.5$ & $71.7 \pm 7.3$ \\
\hline & $\mathrm{CON}$ & FWR & $103.4 \pm 10.8$ & $111.3 \pm 14.0$ & $112.7 \pm 12.5$ & $103.3 \pm 12.1$ & $103.1 \pm 9.6$ \\
\hline & & VR & $115.9 \pm 9.9$ & $118.5 \pm 10.7$ & $110.8 \pm 11.7$ & $100.3 \pm 7.9$ & $99.7 \pm 7.6$ \\
\hline
\end{tabular}

Acronyms: $\mathrm{VL}=$ vastus lateralis; $\mathrm{VM}=$ vastus medialis; Glut = gluteus maximum; $\mathrm{EMG}=$ electromyogram; $\mathrm{MVC}=$ maximum voluntary contraction; FWR = free-weight resistance; $\mathrm{VR}=$ variable resistance; $\mathrm{ECC}=$ eccentric $; \mathrm{CON}=$ concentric. 
Post FWR
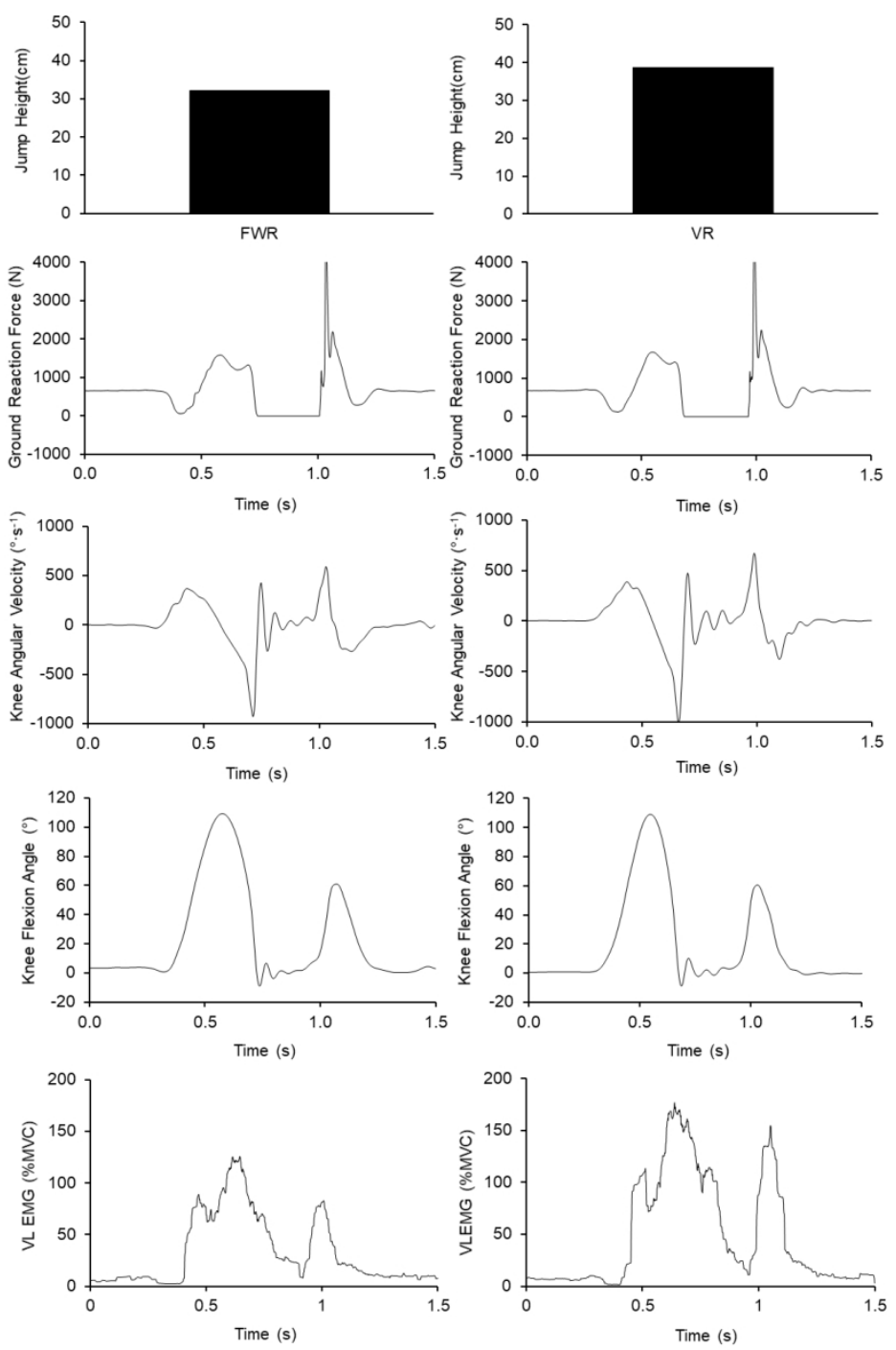

$300 \times 500 \mathrm{~mm}(96 \times 96 \mathrm{DPI})$ 


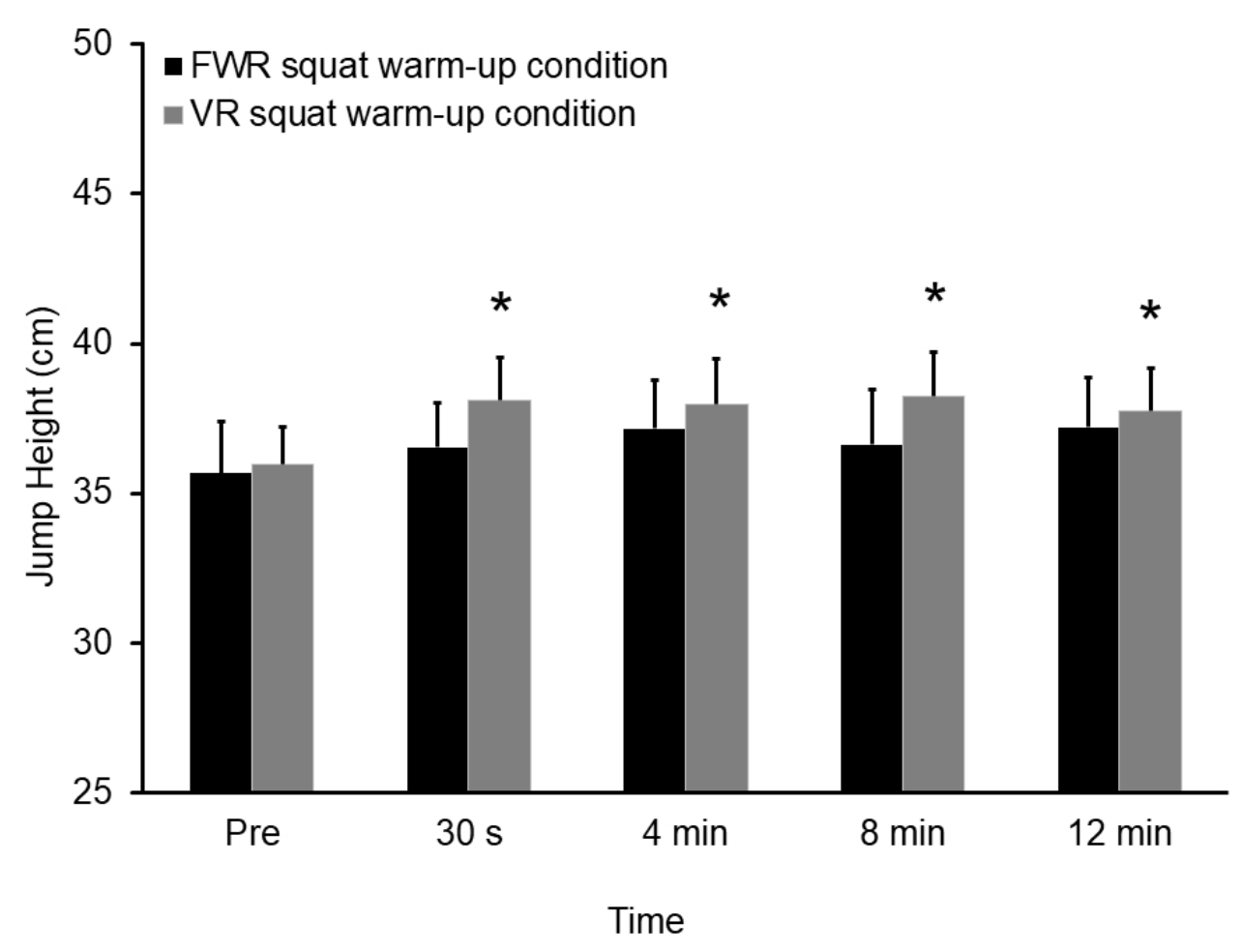

$250 \times 200 \mathrm{~mm}(96 \times 96$ DPI) 